\title{
Neuroprotection - STAIR-Way to the Future?
}

\author{
Ashfaq Shuaib \\ University of Alberta, Edmonton, Canada
}

\author{
Key Words \\ NXY-059 - Acute ischaemic stroke - Neuroprotection • \\ Preclinical development $\cdot$ Primate - Stroke Therapy \\ Academic Industry Roundtable (STAIR)
}

\begin{abstract}
Preclinical testing of putative neuroprotective agents for the treatment of acute ischaemic stroke is critical in assessing their therapeutic potential. Guidelines on how preclinical development of neuroprotectants should be performed were lacking until the Stroke Therapy Academic Industry Roundtable (STAIR) published their recommendations based on lessons from previous failed clinical trials of neuroprotective agents. The STAIR recommendations seek to improve the quality of preclinical research and to ensure that the data generated will enable the selection of those agents most suitable for progression from the laboratory into clinical trials. NXY-059 is a novel free-radical trapping neuroprotectant that is being investigated for the treatment of acute ischaemic stroke. Its preclinical development has closely adhered to the stringent STAIR criteria. Data from independent studies using rodent models of transient middle cerebral artery occlusion (tMCAO) and permanent MCAO (pMCAO) show that NXY-059 reduces infarct volume, improves neurological functioning and has an extended therapeutic window ( $\geq 4 \mathrm{~h}$ ) compared with other agents in late-stage clinical development to date. A key difference in its development compared with previous neuro-
\end{abstract}

protectants is that, in accordance with STAIR criteria, its neuroprotective efficacy has been confirmed in large animal models of pMCAO. These studies in marmosets clearly demonstrate that NXY-059 reduces the functional disability arising from cerebral ischaemia. As functional outcome (rather than infarct volume) is the relevant outcome against which stroke drugs are evaluated in clinical trials, this finding is both important and encouraging. NXY-059 is currently being evaluated in Phase III trials.

Copyright $(2006$ S. Karger AG, Basel

\section{Introduction}

Acute ischaemic stroke is a life-shattering event and is the leading cause of adult disability in industrialised countries [1]. It is also a condition for which safe and effective treatment is urgently needed [2]. Improved understanding of the pathophysiology of acute ischaemic stroke has fuelled interest in neuroprotection as a potential intervention. The goal of neuroprotective strategies is to rescue ischaemically threatened - but potentially viable - brain tissue surrounding the core of infarcted tissue [3] and to attenuate many of the clinical sequelae of stroke, including motor disability and spatial hemineglect. Key agents currently in development include citicoline, a cell-membrane stabiliser, traxoprodil, an NR2B selective $\mathrm{N}$-methyl- $D$-aspartate (NMDA) antagonist, DPb99, a metal ion chelator, and NXY-059, a novel free-

\section{KARGER \\ Fax +4161306 1234 E-Mail karger@karger.ch} www.karger.com
(C) 2006 S. Karger AG, Basel

$1015-9770 / 06 / 0227-0010 \$ 23.50 / 0$

Accessible online at: www.karger.com/ced
Ashfaq Shuaib

2E3.13 Walter Mackenzie Center

University of Alberta

Edmonton, Alberta T6G 2B7 (Canada)

Tel. +1 780407 6395, Fax +1 780407 1325, E-Mail ashfaq.shuaib@ualberta.ca 
radical trapping neuroprotectant that reduces infarct size and preserves brain function in animal models of acute ischaemic stroke [4-7].

The development of neuroprotective therapy for acute ischaemic stroke is challenging. Although a large number of neuroprotective interventions have demonstrated efficacy in preclinical studies involving small animals, clinical studies have so far failed to demonstrate similar efficacy $[8,9]$. This article presents the points that have been learned from evaluating why the success seen in animal models with previous neuroprotective agents did not translate to clinical trials. It will also review the Stroke Therapy Academic Industry Roundtable (STAIR) criteria, which were conceived to optimise the development of new, clinically effective acute stroke treatments; these will be discussed in the context of the preclinical development of NXY-059, which is currently in Phase III clinical trials [10]. In addition, this paper highlights the importance of including primate models as an integral component of the preclinical assessment of agents under investigation for use in acute ischaemic stroke.

\section{Key Lessons from Early Preclinical Investigations}

Methodologies relating to preclinical development may have contributed to the failure of earlier neuroprotective agents to demonstrate success in clinical trials. Many drug development programmes have moved directly from small animal models into clinical trials without intermediary investigation in animal models more relevant to man, which may have caused errors in dose selection. As the pharmacokinetics and pharmacodynamics of neuroprotectants may vary considerably among species, it may not be appropriate simply to scale up the dose used in rodents for use in humans. Clinical trials have consistently failed to match the degree of drug exposure, dose and subsequent plasma levels required for neuroprotection. Several neuroprotective agents have been investigated in clinical trials at doses that were neuroprotective during preclinical assessment, despite causing adverse events in rodents, thus inevitably leading to intolerance and possibly adverse events in man and preventing detection of efficacy [11]. With other agents, adverse events have limited the opportunity to administer them at doses that have demonstrated neuroprotection in animals [12]. Others may have demonstrated efficacy in animal models of transient ischaemia but not in clinical trials, because adverse events prevented the achievement of the higher doses needed for neuroprotection in permanent models of ischaemia [13, 14].

Additionally, many agents have been administered beyond the therapeutic time window of opportunity determined in preclinical animal models. For example, despite preclinical evidence suggesting that NMDA receptor antagonists act in the early part of the ischaemic cascade [3] and are active for 60-90 minutes post-occlusion [15], in clinical trials these agents were administered around $6 \mathrm{~h}$ after onset of stroke [16]. In contrast, clinical trials have shown recombinant tissue plasminogen activator (rt-PA) to be effective within a 3-hour window post-stroke [17], which is consistent with the timeframe determined in animal studies [18]. Clearly, as very short treatment time windows are a challenge in clinical practice, the therapeutic window of opportunity determined at the preclinical development stage has a major impact on the development of current neuroprotectants. The rt-PA data emphasise that clinical trials should evaluate novel compounds using time windows that are comparable to those in animals.

Many early neuroprotectants had not been studied appropriately in animal models with a view to their use in humans, which contributed to their failure. Although damage to white matter is involved in acute ischaemic stroke in humans [19], few agents were evaluated for their ability to protect white matter prior to clinical trials since rodent brains contain only a small amount of white matter, and rodent models formed the basis of preclinical studies [16]. Additionally, some investigations have focused on the agents' level of protection to the penumbra, which is not always present in cases of acute ischaemic stroke in humans. Early preclinical investigations tend to assess neuroprotective efficacy in terms of the histological reduction of infarct volume, with negligible attention to effect on functional recovery [16], which is a major endpoint in clinical trials.

\section{Steps for the Optimal Development of Neuroprotectants}

The plethora of failed clinical trials of previous neuroprotectants has raised the question of how preclinical investigation should best be conducted in the future. Preclinical testing of neuroprotective agents is critical in assessing their therapeutic potential. Previous investigations have concentrated on agents that acted at the early part of the ischaemic cascade (including glutamate antagonists, ion channel compounds, clomethiazole), thus 
providing a short therapeutic window of opportunity. Steps are now being taken to implement strategies that will identify agents more worthy of investigation in clinical trials. Agents currently under investigation have a mode of action targeting the latter part of the ischaemic cascade. The dosages used will produce clinically relevant plasma levels at a time post-infarction that has clinical utility. To ensure that the preclinical data obtained are relevant to the clinical situation, efficacy data have been obtained in rodent models of both transient focal ischaemia and permanent ischaemia. Recent studies have shown that the dose of a neuroprotectant effective in transient models of ischaemia does not necessarily generate sufficient plasma concentrations to provide neuroprotection in permanent models of ischaemia [7, 20]. Of the two models of ischaemia, the permanent model mimics the human clinical situation more closely because many infarcts reperfuse slowly, if at all [21]. Hence, doses of neuroprotectants chosen for investigation in clinical trials should reflect doses that were effective in permanent models of ischaemia. Additionally, primate models of acute ischaemic stroke have been developed [22, 23]. Testing new neuroprotectants in these models will reduce the difficulties of extrapolating findings from rodents to humans and provide evidence for neuroprotection at time durations comparable to the clinical situation. Many of these actions, amongst others, have been incorporated into recommendations based on the learning points derived from the failed trials [24]. The STAIR recommendations aim to improve the quality of preclinical research and to ensure that the data generated will enable the selection of those agents most likely to succeed in appropriately designed clinical trials.

\section{STAIR Recommendations}

The STAIR recommendations [24] make comprehensive reference to the preclinical investigation of novel neuroprotectants. Based on lessons from failed clinical trials of agents evaluated beyond their window of opportunity, e.g. the NMDA antagonists [25], the guidelines emphasise the importance of developing agents that have windows of opportunity of relevance to the clinic. As a majority of stroke patients typically arrive at hospital up to $6 \mathrm{~h}$ after onset of stroke and beyond [25], agents considered for preclinical development should be those that act in the latter stages of the ischaemic cascade and should have an extended therapeutic window of opportunity. The guidelines highlight that the conditions surrounding the selection of the target concentration effective in animal models should be relevant to the clinical situation of stroke in humans. For this reason, STAIR endorses the testing of agents in permanent models of ischaemia, noting that much higher drug exposure seems to be required to provide neuroprotection in models of permanent ischaemia compared with transient models [7]. The importance of testing agents in permanent models is highlighted by the failed trial of clomethiazole [13], in which, because of side effects, the drug could only achieve plasma levels that were effective in a rat model of transient ischaemia. The STAIR guidelines also emphasise the importance of measuring efficacy in terms of both functional improvement and a reduction in infarct size. These outcomes should be determined in both small and large animal models (such as primates and cats), especially for first-in-class therapies. Typical outcome measures include immunohistochemical analysis, neuropathology, somatosensory-evoked potentials, electroencephalography and neurobehaviour. To gain a complete understanding of the drug's effects, outcome measures should be monitored during the acute phase, following drug administration (hours-days) and in the long-term (days-weeks). Functional recovery is highlighted as a key outcome measure as it is a major endpoint of clinical trials and does not always correlate well with infarct size. Although this parameter can be difficult to measure in rodent models of ischaemia due to the animals' high degree of plasticity, evaluation is achievable in primate models of acute ischaemic stroke $[23,26]$ because these models most closely replicate the human response to stroke and its treatment.

\section{NXY-059: A Novel Free Radical-Trapping Neuroprotectant}

Free radicals play a major part in the damage caused by hypoxia and reperfusion during cerebral ischaemia [27]. NXY-059 is a novel free radical-trapping neuroprotectant. It is currently being investigated for the treatment of acute ischaemic stroke and its preclinical development adhered to the STAIR criteria.

\section{NXY-059 in Small-Animal Models of Permanent and}

Transient Middle Cerebral Artery Occlusion (MCAO)

The neuroprotective efficacy of NXY-059 was initially demonstrated in a series of experiments in small-animal models of transient focal ischaemia [4, 7]. Dose-response studies in the rodent transient middle cerebral 
artery occlusion (tMCAO) model showed that NXY-059 $0.3-30 \mathrm{mg} / \mathrm{kg}$ for loading and a continuous infusion of $0.3-30 \mathrm{mg} / \mathrm{kg} / \mathrm{h}$ for $24 \mathrm{~h}$ reduced infarct volume in a dosedependent manner, as measured on the second day after occlusion [4]. Additionally, dose-dependent improvements in neurological symptoms were also noted at 24 and $48 \mathrm{~h}$ post-ischaemia. These findings were supported by the observations of a second study [7]. Intravenous infusion of NXY-059 at doses of $1-30 \mathrm{mg} / \mathrm{kg} / \mathrm{h}$ for $21.75 \mathrm{~h}$, starting $2.25 \mathrm{~h}$ after the occlusion, resulted in decreased infarct volume (10 and $30 \mathrm{mg} / \mathrm{kg}$ only) and dose-dependent reductions in neurological impairment (all doses tested) [7]. The efficacy observed by Kuroda et al. at lower doses $(<10 \mathrm{mg} / \mathrm{kg})$ may have been due to the initial loading dose in the study, which was not used by the Sydserff group. The similar findings from both of these studies suggest that NXY-059 provides substantial neuroprotection in terms of both reduced infarction and recovery from neurological deficits.

In accordance with STAIR recommendations [24], the neuroprotective efficacy of NXY-059 has also been demonstrated in animal models of permanent focal ischaemia, which simulate the conditions of human stroke more closely than transient models. A major study of permanent MCAO (pMCAO) [7] assessed NXY-059 at loading doses of $30-70 \mathrm{mg} / \mathrm{kg}$ followed by a 24-hour infusion of $30-70 \mathrm{mg} / \mathrm{kg}$. When treatment was administered $5 \mathrm{~min}$ post-occlusion, neuroprotection was demonstrated in a dose-dependent manner at all doses tested [7]. Protection was demonstrated not only when measured as total volume of damage, but also when assessed in terms of cortical and subcortical damage. A linear relationship was observed between dose, plasma concentration and neuroprotection. Extrapolation of these data suggests that $\sim 80 \%$ neuroprotection may be possible when the plasmafree concentration of NXY-059 is $\sim 150 \mu \mathrm{mol} / 1$ at $24 \mathrm{~h}$ of infusion [7], which meets the level of protection suggested as sufficient to justify proceeding to a clinical trial [28]. Similar dose-dependent neuroprotective effects of NXY-059 were demonstrated in a different model of permanent focal ischaemia [20]. Infusion of NXY-059 at doses of 30 or $60 \mathrm{mg} / \mathrm{kg}$ for $24 \mathrm{~h}, 5 \mathrm{~min}$ post-occlusion, resulted in a modest decrease in cortical damage at the lower doses and significant protection at the higher dose [20].

The data from these studies with NXY-059 suggest that higher drug exposure is needed to provide protection in models of permanent compared with transient ischaemia. This observation may be key to the pharmaceutical development of clinically efficacious neuroprotectants and supports the recommendation by STAIR that novel neuroprotectants should demonstrate efficacy in both transient and permanent models of ischaemia before they progress into clinical trials [24].

An additional experiment in a transient model of ischaemia confirmed that NXY-059 produced long-term neuroprotection rather than merely delaying the appearance of damage [4]. Data showed that the degree of neuroprotection with NXY-059 was similar at 2 and 7 days postischaemia. Moreover, when the agent was infused for $48 \mathrm{~h}$ at $30 \mathrm{mg} / \mathrm{kg} / \mathrm{h}$ after $2 \mathrm{~h}$ of MCAO, a marked reduction in infarct volume, compared to the control group, was also observed on Day 7 after ischaemia, indicating that NXY-059 has prolonged effects.

Further experiments in models of tMCAO and pMCAO explored the therapeutic window of NXY-059. In the tMCAO model, NXY-059 was found to substantially reduce infarct volume when administered $5 \mathrm{~h}$ after ischaemia [4]. Similarly, a reduction in ischaemic damage was observed with NXY-059 in pMCAO when administered up to $4 \mathrm{~h}$ post-occlusion (fig. 1) [7]. The data from these studies suggest that the therapeutic window of NXY-059 is sufficient to be clinically relevant.

Overall, these data indicate that NXY-059 is effective in rodents, with a time window that is achievable in clinical practice and at plasma concentrations that can be achieved in humans.

\section{NXY-059 in the Marmoset Model of pMCAO}

A key difference in the development of NXY-059 compared with earlier neuroprotective agents is that, in accordance with STAIR criteria, the neuroprotective efficacy demonstrated in rodent models has been confirmed in large animal pMCAO models. The marmoset was selected as the large animal model for stroke for evaluation of NXY-059 because it has several advantages over other models such as rodents or cats; marmosets are phylogenetically close to man, provide an opportunity to model higher mental functions (including behaviour) and have a large brain-to-body size ratio [22].

The efficacy of NXY-059 in reducing the long-term functional disability and damage arising from cerebral ischaemia was evaluated in two studies using the marmoset pMCAO model $[5,6]$. In both studies the functional outcome was measured using the Hill and Valley tests. Examining the use of each arm reaching into hemispace allows the effects of unilateral motor impairment, confined to the left arm to be dissociated from unlilateral perceptual spatial impairment, confined to the left hemispace. 
Fig. 1. Time window of neuroprotection with NXY-059 in rats in permanent and transient ischaemia (data recalculated from Kuroda et al. [4] and Sydserff et al. [7]).

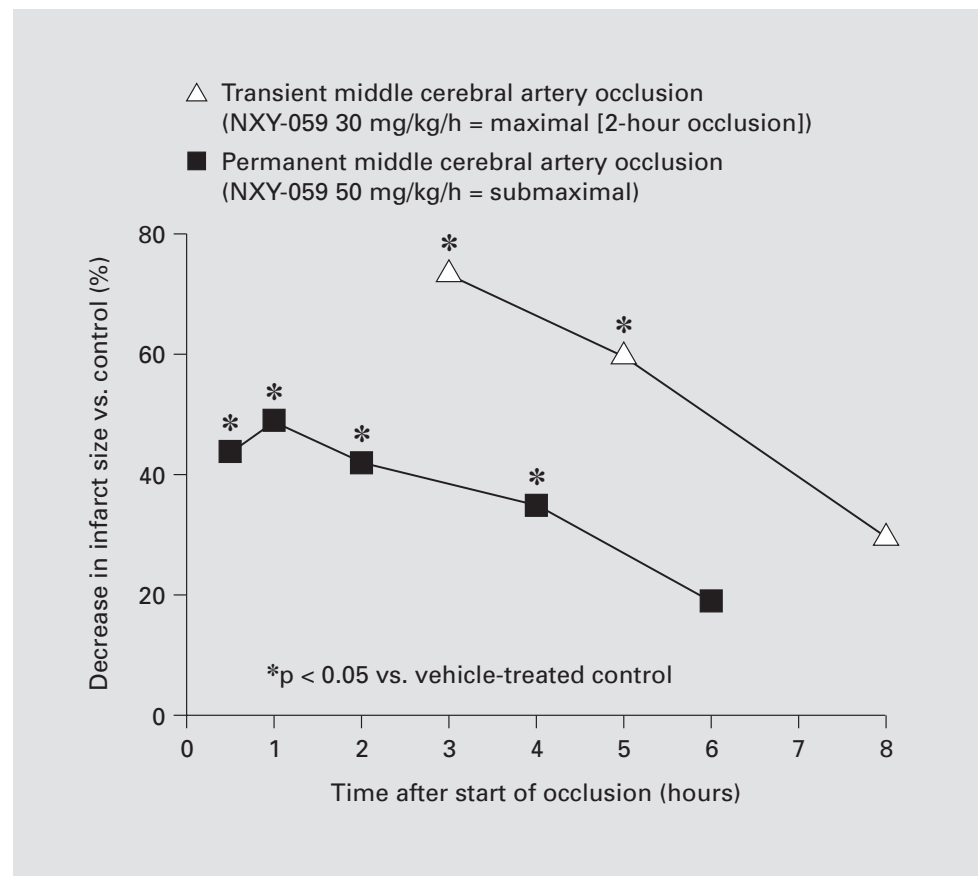

In the first study [5], 12 marmosets received a loading dose of either NXY-059 $(28 \mathrm{mg} / \mathrm{kg})$ or saline control 5 min after onset of ischaemia, followed by continuous infusion $(16 \mathrm{mg} / \mathrm{kg} / \mathrm{h})$ or saline for $48 \mathrm{~h}$. After $24 \mathrm{~h}$ of treatment, the plasma unbound drug concentration of NXY-059 was $76.3 \mu \mathrm{mol} / \mathrm{l}$. The main finding from this study was that NXY-059 lessened functional disability in the marmoset model of pMCAO. In the Hill and Valley tests, NXY-059-treated marmosets were significantly better than those treated with saline at reaching with their affected arm at 3 weeks $(p<0.01)$ and 10 weeks $(p<0.01)$ after surgery. At these assessments, four of the six marmosets in the NXY-059 group had near-normal motor performance. These observations show that NXY-059 reduced long-term disability and that the benefit seen with NXY-059 after infarct is due to true neuroprotection rather than to a delay in the appearance of damage. A second key finding was that NXY-059 treatment significantly reduced the degree of spatial perceptual neglect $(p<0.01)$ compared with saline-treated marmosets. This is of clinical relevance because cognitive deficits such as spatial neglect are debilitating and can be a significant barrier to a stroke patient's rehabilitation [29]. Histological analysis showed that treatment with NXY-059 reduced the volume of damage by $>50 \%$ compared with controls. Further histological assessment of the condition of the individual structures of the brain, which had not previously been possible in rodent models of stroke, showed that protection was not only evident in the cortex but also extended to the white matter, caudate and putamen, demonstrating that NXY-059 prevents damage to structures of the brain that are at risk in human stroke [5].

Based on these positive findings, a second study [6] was conducted to investigate the efficacy of NXY-059 at the more clinically relevant time of $4 \mathrm{~h}$ after occlusion in the same pMCAO model in marmosets. Four hours after pMCAO, the marmosets were allocated in a blinded manner to NXY-059 (77 $\mu \mathrm{mol} / \mathrm{kg}$ i.v. plus $154 \mu \mathrm{mol} / \mathrm{kg}$ subcutaneous injection) or saline control followed by a further 48 -h i.v. infusion of NXY-059 $(85 \mu \mathrm{mol} / \mathrm{kg} / \mathrm{h})$ or saline. The dose of NXY-059 used in this study was higher than that used in the earlier study and was selected to produce plasma concentrations that were considerably above those by then shown to be neuroprotective in rodent models of pMCAO and similar to plasma levels known to be safely tolerated in human stroke patients. The mean plasma unbound drug concentration achieved was $200 \mu \mathrm{mol} / 1$, which is $75 \%$ of the plasma levels found to be well tolerated by stroke patients in a clinical trial (260 $\mu \mathrm{mol} / \mathrm{l})$ [30]. In the Hill test, NXY-059-treated marmosets were significantly better at reaching with their affected left arm into neglected left hemispace compared with the control group ( 3 weeks, $\mathrm{p}<0.05 ; 10$ weeks, $\mathrm{p}<$ 
Fig. 2. Average scores achieved by NXY-059-treated and saline-control marmosets on the Valley staircase test. Data shown are tests with each arm 3 and 10 weeks after permanent middle cerebral artery occlusion (pMCAO). Four hours after pMCAO, the marmosets received NXY-059 (77 $\mu \mathrm{mol} / \mathrm{kg}$ i.v. plus $154 \mu \mathrm{mol} / \mathrm{kg}$ subcutaneous injection) or saline control followed by a further 48-h i.v. infusion of NXY-059 $(85 \mu \mathrm{mol} / \mathrm{kg} / \mathrm{h})$ or saline [6].
Fig. 3. Average scores of use of the left arm achieved by NXY-059-treated and saline-control marmosets in the Valley staircase test. Data show use of the left arm 3 and 10 weeks after permanent middle cerebral artery occlusion (pMCAO). Four hours after pMCAO, the marmosets received NXY-059 $(77 \mu \mathrm{mol} / \mathrm{kg}$ i.v. plus $154 \mu \mathrm{mol} / \mathrm{kg}$ subcutaneous injection) or saline control followed by a further 48-h i.v. infusion of NXY-059 $(85 \mu \mathrm{mol} / \mathrm{kg} / \mathrm{h})$ or saline [6].
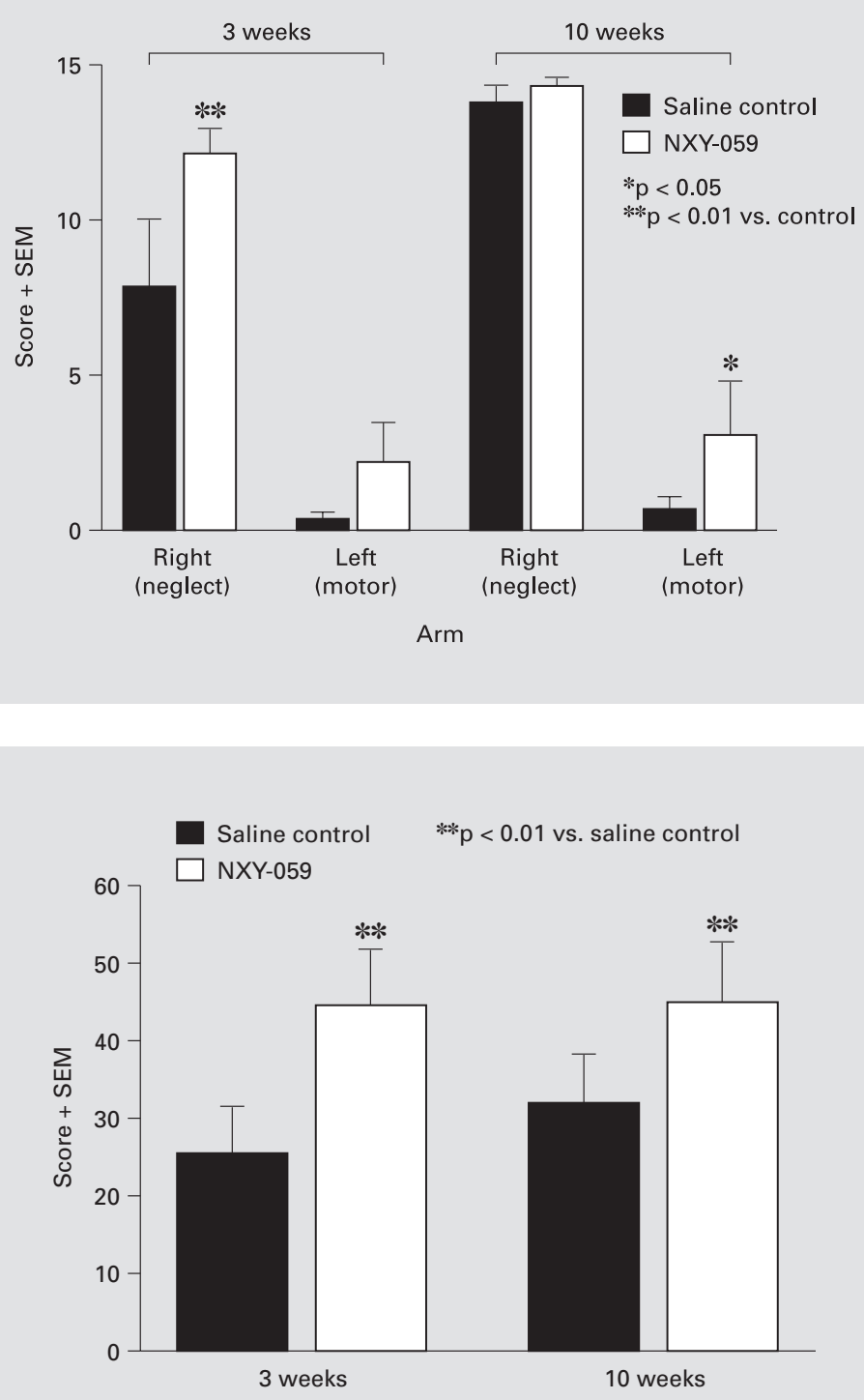

0.01). While the deficit in using the affected left arm on this task reflects both a spatial neglect and motor problem, as other tests had shown spatial neglect to be negligible by 10 weeks, these data suggest that the left arm use in NXY-059-treated animals at 10 weeks primarily reflects an improvement in motor function. In the Valley test, there was no significant difference in reaching with the affected left arm into right unneglected (motor) hemispace at 3 weeks, but there was a clear effect at 10 weeks ( $<<0.05$; fig. 2). At 3 weeks, NXY-059-treated marmosets were significantly better than control animals at reaching with their unaffected right arm into left ne- glected hemispace on the Valley test $(p<0.01$; fig. 2$)$. At 10 weeks, both the NXY-059 and control groups could reach into left neglected hemispace with their unaffected right arm without any significant deficit (fig. 2). As the Valley test measures the use of the affected left arm into the right hemispace, these data add credence to the proposal that NXY-059 improves motor function. In both the Hill and Valley tests, NXY-059-treated marmosets demonstrated a significantly greater use of the affected left arm at 3 weeks and at 10 weeks $(p<0.01)$, which further supports a role for NXY-059 in restoring motor function (fig. 3). 
The histological data from this study support the benefits in the functional outcomes observed with NXY-059 in this trial. Quantitative histological analysis of brain tissue was conducted 11 weeks after surgery. Saline-treated animals had substantial damage in the right hemisphere of their brains that extended to subcortical structures, with almost total loss of caudate and putamen. In the NXY-059-treated group, the total infarct volume was $28 \%$ lower than in the controls and less damage was evident in the cortex, the white matter and the caudate and the putamen, although the decrease was only significant for the putamen $(p=0.045)$ [6]. Overall, these data support the histological observations from the earlier primate study [5] and provide convincing evidence that NXY-059 protects both grey and white matter of the brain from damage after acute ischaemic stroke when administered $4 \mathrm{~h}$ after the start of pMCAO.

\section{Summary}

Lessons learned from the failure of clinical trials of early neuroprotective agents in acute ischaemic stroke have been captured in the STAIR guidelines for the preclinical assessment of new agents. These criteria provide recommendations for a rigorous, robust and detailed preclinical evaluation of neuroprotective drugs. Adherence to these guidelines should lead to the identification of agents that have a reasonable chance of succeeding in appropriately designed clinical trials.
NXY-059 has undergone preclinical development as directed by the STAIR recommendations. In models of acute ischaemic stroke in two species, NXY-059 decreased infarct volume and reduced long-term disability, suggesting it has neuroprotective effects. Furthermore, efficacy was achievable within a time window relevant to clinical practice. A key difference in the development of NXY-059 compared with some of the earlier neuroprotectants is that, in accordance with STAIR criteria, its neuroprotective efficacy has been demonstrated in a primate model of acute ischaemic stroke. Evidence of the neuroprotective effect of NXY-059 in the marmoset pMCAO model of long-term disability caused by ischaemic stroke is both important and encouraging. Following the positive findings from the preclinical investigations, NXY-059 is currently in late-stage clinical development. As the first neuroprotective agent to have progressed to Phase III clinical trials (SAINT I [10] and SAINT II) on the basis of having satisfied all the STAIR preclinical criteria, there is much interest in the outcome from these trials with NXY-059.

\section{Acknowledgement}

I thank Maxine Holland from Complete Medical Group, who provided medical writing support on behalf of AstraZeneca.

\section{References}

1 World Health Organization: Statistical Information System. World Health Organization 2004.

2 Fisher M, for the Stroke Therapy Academic Industry Roundtable: Recommendations for advancing development of acute stroke therapies. Stroke Therapy Academic Industry Roundtable 3. Stroke 2003;34:1539-1546.

-3 Dirnagl U, Iadecola C, Moskowitz MA: Pathobiology of ischaemic stroke: an integrated view. Trends Neurosci 1999;22:391-397.

4 Kuroda S, Tsuchidate R, Smith ML, Maples KR, Siesjö BK: Neuroprotective effects of a novel nitrone, NXY-059, after transient focal cerebral ischemia in the rat. $\mathrm{J}$ Cereb Blood Flow Metab 1999;19:778-787.

5 Marshall JW, Duffin KJ, Green AR, Ridley RM: NXY-059, a free radical-trapping agent, substantially lessens the functional disability resulting from cerebral ischemia in a primate species. Stroke 2001;32:190-198.
Marshall JWB, Cummings RM, Bowes LJ, Ridley RM, Green AR: Functional and histological evidence for the protective effect of NXY-059 in a primate model of stroke when given $4 \mathrm{~h}$ after occlusion. Stroke 2003;34: 2228-2233.

7 Sydserff SG, Borelli AR, Green AR, Cross AJ: Effect of NXY-059 on infarct volume after transient or permanent middle cerebral artery occlusion in the rat; studies on dose, plasma concentration and therapeutic time window. Br J Pharmacol 2002;135:103-112.

8 Kidwell CS, Liebeskind DS, Starkman S, Saver JL: Trends in acute ischemic stroke trials through the 20th century. Stroke 2001;32: 1349-1359.

-9 Ovbiagele B, Kidwell CS, Starkman S, Saver JL: Neuroprotective agents for the treatment of acute ischemic stroke. Curr Neurol Neurosci Rep 2003;3:9-20.
10 Lees KR, Zivin JA, Ashwood T, Davalos A, Davies SM, Diener HC, Grotta J, Lyden P, Shuaib A, Hardemark HG, Wasiewski WW: NXY-059 for acute ischemic stroke. N Engl J Med 2006;354:588-600.

11 Dawson DA, Wadsworth G, Palmer AM: A comparative assessment of the efficacy and side-effect liability of neuroprotective compounds in experimental stroke. Brain Res 2001;892:344-350.

12 Muir KW, Lees KR: Clinical experiences with excitatory amino acid antagonists. Stroke 1995;26:503-513.

13 Lyden P, Shuaib A, Ng K, Levin K, Atkinson RP, Rajput A, Wechsler L, Ashwood T, Claesson L, Odergren T, Salazar-Grueso E: Clomethiazole Acute Stroke Study in ischemic stroke (CLASS-I): final results. Stroke 2002;33:122128. 
14 Sydserff SG, Cross AJ, Murray TK, Jones JA, Green AR: Clomethiazole is neuroprotective in models of global and focal cerebral ischemia when infused at doses producing clinically relevant plasma concentrations. Brain Res 2000; 862:59-62.

15 Massieu L, Thedinga KH, McVey M, Fagg GE: A comparative analysis of the neuroprotective properties of competitive and uncompetitive $\mathrm{N}$-methyl-D-aspartate receptor antagonists in vivo: implications for the process of excitotoxic degeneration and its therapy. Neuroscience 1993; 55:883-892.

16 Green AR, Odergren T, Ashwood T: Animal models of stroke: do they have value for discovering neuroprotective agents? Trends Pharmacol Sci 2003;24:402-408.

17 NINDS rt-PA Stroke Study Group: Tissue plasminogen activator for acute ischemic stroke. The National Institute of Neurological Disorders and Stroke rt-PA Stroke Study Group. N Engl J Med 1995;333:1581-1587.

18 Brinker G, Franke C, Hoehn M, Uhlenkuken U, Hourihane JM: Thrombolysis of cerebral clot embolism in rat: effect of treatment delay. NeuroReport 1999;10:3269-3272.
19 Dewar D: Drug development for stroke: importance of protecting cerebral white matter Eur J Pharmacol 1999;375:41-50.

20 Zhao Z, Cheng M, Maples KR, Ma JY, Buchan AM: NXY-059, a novel free radical trapping compound, reduces cortical infarction after permanent focal cerebral ischemia in the rat Brain Res 2001;909:46-50.

21 Ringelstein EB, Biniek R, Weiller C, Ammeling B, Nolte PN, Thron A: Type and extent of hemispheric brain infarctions and clinical outcome in early and delayed middle cerebral artery recanalization. Neurology 1992;42:289298.

22 Marshall JW, Ridley RM: Assessment of functional impairment following permanent middle cerebral artery occlusion in a non-human primate species. Neurodegeneration 1996;5: 275-286.

23 Marshall JW, Cross AJ, Ridley RM: Functional benefit from clomethiazole treatment after focal cerebral ischaemia in non-human primate species. Exp Neurol 1999;156:121-129.

24 Stroke Therapy Academic Industry Roundtable (STAIR): Recommendations for standards regarding preclinical neuroprotective and restorative drug development. Stroke 1999;30: 2752-2758.
25 Davis SM, Lees KR, Albers GW, Diener HC, Markabi S, Karlsson M, Norris J: Selfotel in acute ischemic stroke: possible neurotoxic effects of an NMDA antagonist. Stroke 2000;31: 347-354.

26 Marshall JW, Ridley RM: Assessment of cognitive and motor deficits in a marmoset model of stroke. ILAR J 2003;44:153-160.

27 Siesjo BK, Agardh CD, Bengtsson F: Free radical and brain damage. Brain Metab Rev 1989; $1: 165-211$.

28 Grotta J: Neuroprotection is unlikely to be effective in humans using current trial designs. Stroke 2002;33:306-307.

29 Robertson IH, Halligan PW, Marshall JC: Prospects for the rehabilitation of unilateral neglect; in Robertson IH, Marshall JC (eds): Unilateral Neglect: Clinical and Experimental studies. Hove, UK, Lawrence Erlbaum Associates Ltd, 1993.

30 Lees KR, Barer D, Ford GA, Hacke W, Kostulas V, Sharma AK, Odergren T, for the SANXY-0004 Investigators: Tolerability of NXY-059 at higher target concentrations in patients with acute stroke. Stroke 2003;34: 482-487. 